\title{
RECONSTRUCTION OF THE EXTENSOR APPARATUS OF THE KNEE WITH THE LEEDS-KEIO LIGAMENT
}

\author{
K. FUJIKAWA, T. OHTANI, H. MATSUMOTO, B. B. SEEDHOM
}

From Keio University, Japan

We describe a new method for the reconstruction of ruptures of the patellar ligament or the quadriceps tendon, using the flexible open-weave polyester LeedsKeio prosthetic ligament. Of 25 operations performed since 1985, we were able to review 18 patients (19 knees) with a mean follow-up of 3.5 years ( 3 to 6.5).

No patient had required immobilisation of the knee after operation and the average period to return to normal activities without a walking aid was 10.5 weeks. The average range of motion was $146^{\circ}$, and four patients could squat fully in the Japanese style. There was an extension lag $\left(<10^{\circ}\right)$ in only four patients; eight patients had some patellar crepitus which was mildly painful in three. There were no cases of infection, persistent joint effusion or rerupture of the extensor apparatus.

J Bone Joint Surg [Br] 1994; 76-B:200-3.

Received 16 March 1993; Accepted 17 June 1993

Rupture of the extensor apparatus of the knee is an uncommon but severe injury which has a long recovery period and often leaves an extension lag and limitation of flexion. No previous surgical procedure has allowed early return to activity and full function.

We report a new operation for the reconstruction of the knee extensor apparatus using the Leeds-Keio artificial ligament.

\section{PATIENTS AND METHODS}

The new procedure has been performed on 25 knees in 24 patients since February 1985 . There were 17 men and seven women; their average age was 37.5 years. In 12 knees (11 patients) pathological rupture was secondary to renal failure;

K. Fujikawa, MD, MPhil (Bioeng), Associate Professor

T. Ohtani, MD, Instructor in Orthopaedic Surgery

H. Matsumoto, MD, PhD (Bioeng), Instructor in Orthopaedic Surgery

Department of Orthopaedic Surgery, School of Medicine, Keio University, 35 Shinanomachi, Shinjuku-ku, Tokyo, Japan.

B. B. Seedhom, BSc, PhD, ARC Senior Lecturer in Bioengineering Rheumatology and Rehabilitation Research Unit, University of Leeds, 36 Clarendon Road, Leeds LS2 9NZ, UK.

Correspondence should be sent to Dr B. B. Seedhom.

C1994 British Editorial Society of Bone and Joint Surgery $0301-620 X / 94 / 2688 \$ 2.00$ five patients had a quadriceps tendon rupture, six had a patellar tendon rupture and one had both. In five knees there was nonunion of a displaced patellar fracture, five had had open comminuted fractures or patellectomy with severe arthrofibrosis, and two had patellar tendon rupture during professional sport. One knee had patellar tendon rupture after total knee replacement.

Implant and techniques. The tube-type Leeds-Keio artificial ligament is made of polyester fibre as an open-weave mesh with rectangular holes 1.5 to $2.5 \mathrm{~mm}^{2}$ in area. It is designed to act as a scaffold for invasion by soft tissue, which will mature and develop well-aligned fibres capable of sharing the load with the implant. The implant is $60 \mathrm{~cm}$ in length (Fig. 1), and has a maximum tensile strength of about $2200 \mathrm{~N}$ with a stiffness of $195 \mathrm{kN} / \mathrm{m}$ at a strain rate of $1 \%$ and $279 \mathrm{kN} / \mathrm{m}$ at $100 \%$ per second.

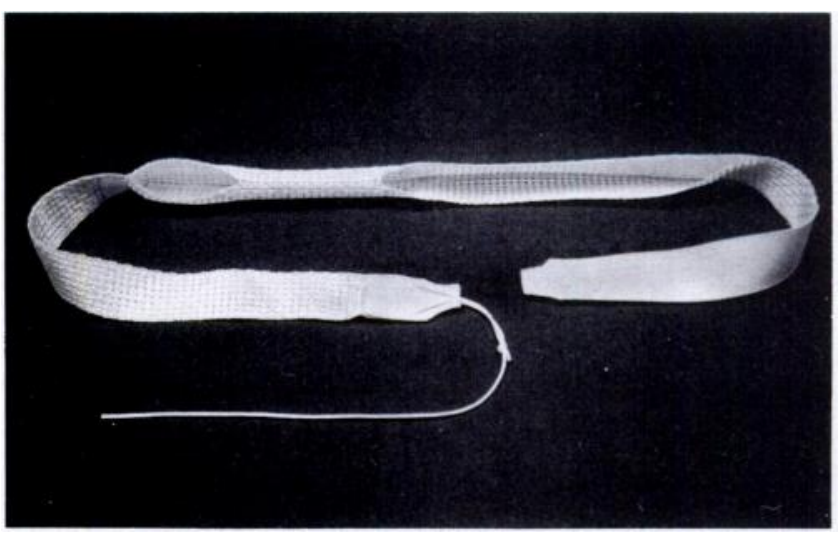

Fig. 1

The tubular Leeds-Keio artificial ligament.

Patellar tendon reconstruction. An anteromedial longitudinal incision exposes the distal end of the quadriceps tendon and tibial tuberosity. The remnants of the ruptured tendon are preserved. The artificial ligament is passed transversely through the distal end of the quadriceps tendon along the proximal margin of the patella and is brought down distally to cross itself in front of the patella as a figure-of-eight. One end of the implant is then passed through a transverse tunnel drilled through the tibia $1 \mathrm{~cm}$ posterior to the tibial tuberosity. Finally, both ends are anchored to the lateral tibial condyle under the tibialis anterior muscle by double staples. The artificial ligament is pulled tight with the knee flexed to $20^{\circ}$, 

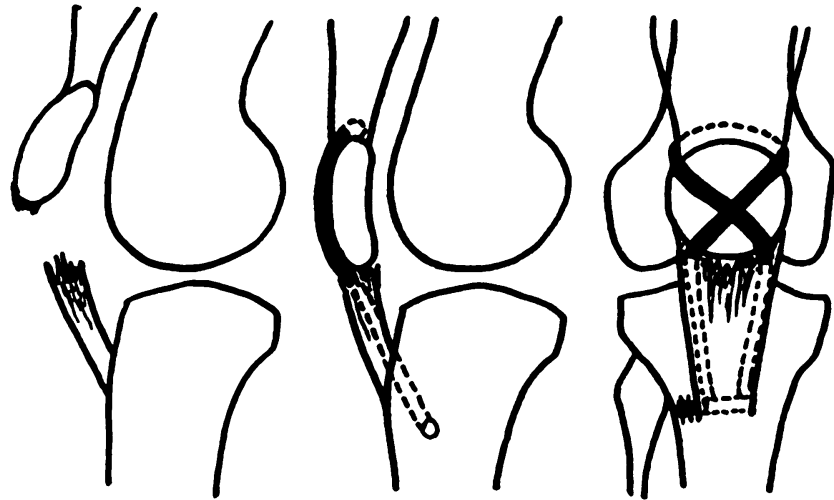

Fig. 2

Patellar tendon reconstruction with the Leeds-Keio artificial ligament.

bringing the distal articular surface of the patella into contact with the femur (Fig. 2). The remnants of the original patellar tendon are sutured to the implant. This technique restores the anatomical relationship, and preserves both the patellofemoral congruity and normal tracking of the patella.

Quadriceps tendon reconstruction. The artificial ligament is placed transversely through the proximal end of the patellar tendon close to the inferior border of the patella, crossed over the patella and crossed again through the proximal quadriceps tendon. The ends of the implant are sutured to each other and into the quadriceps femoris muscle group (Fig. 3).

Postoperative management. The knee is not splinted; continuous passive motion from $0^{\circ}$ to $90^{\circ}$ is started one day after the operation. After one week the range of motion is increased to the limit of the patient's tolerance. After patellar
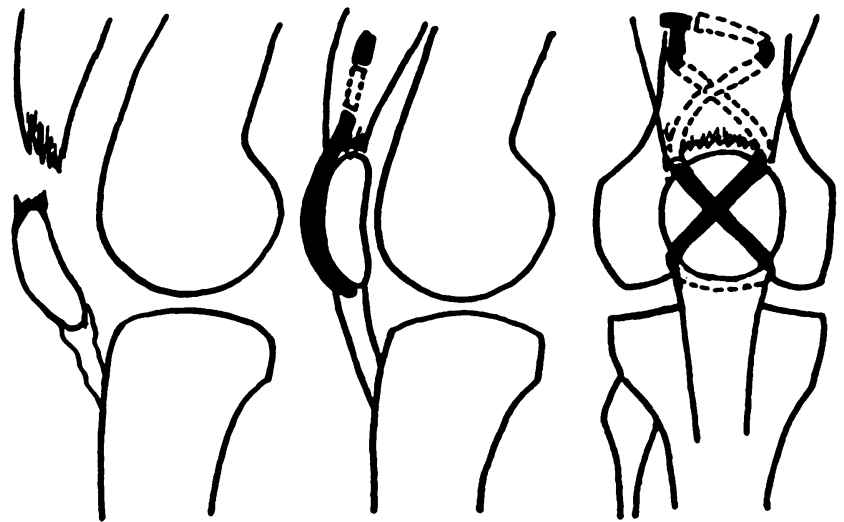

Fig. 3

Quadriceps tendon reconstruction with the Leeds-Keio artificial ligament.

tendon reconstruction partial weight-bearing in a brace is permitted at two weeks; after quadriceps tendon reconstruction this starts at four weeks. Full weight-bearing is allowed after three weeks and six weeks respectively.

\section{RESULTS}

Six patients were lost to follow-up. The patient with patellar tendon rupture after a total knee replacement had an extension lag of $5^{\circ}$ and could flex to $87^{\circ}$. The other 18 patients (19 knees) were reviewed after 3 to 6.5 years (mean 42 months). The average range of knee movement was $146.4^{\circ}$ (Table I). Four of the patients could squat fully in the Japanese style. Three had an extension lag of less than $10^{\circ}$. Eight patients complained of patellar crepitus and three of these had mild pain during normal activities. The average period to return

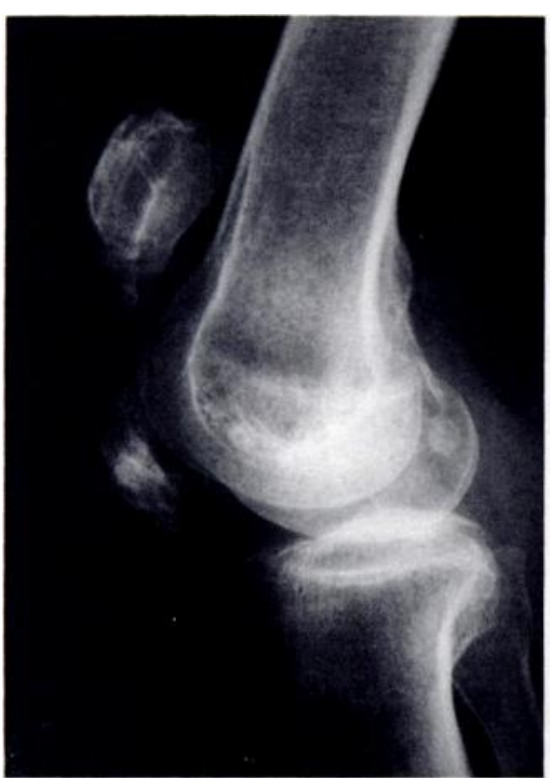

Fig. 4a

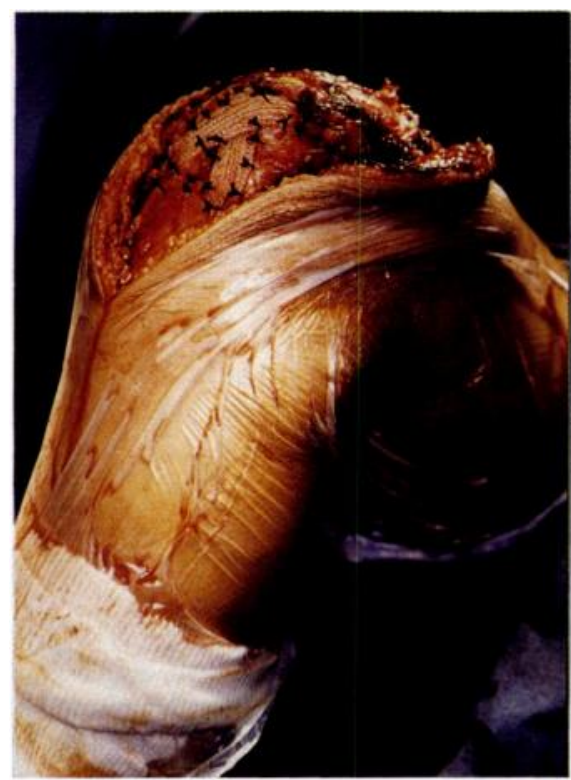

Fig. 4b

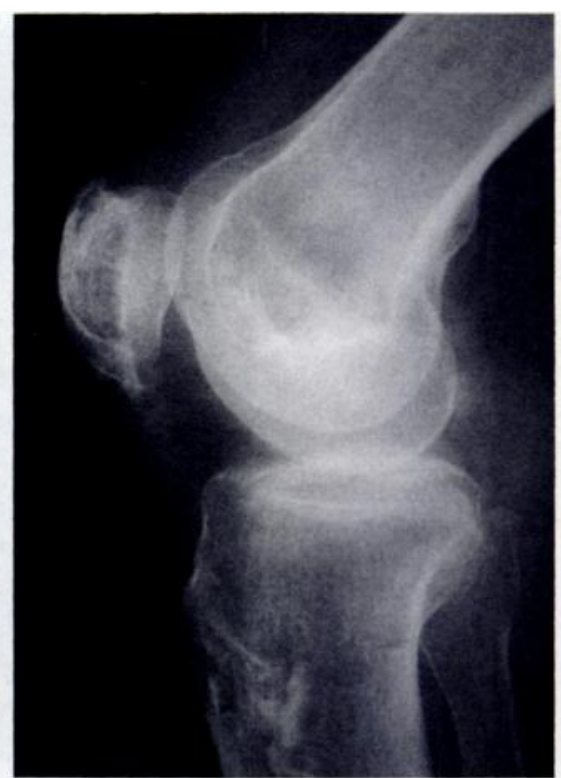

Fig. 4c

Case 1. A 65-year-old man operated on two years after a patellar fracture. Figure 4a - Preoperative radiograph. Figure 4 b - At operation the distal fragment of the patella was excised and the patellar tendon was reconstructed with the artificial ligament to pull the patella down into an anatomical position. Figure $4 c$ - Radiograph five years after repair, when the patient had full flexion and no extension lag. 


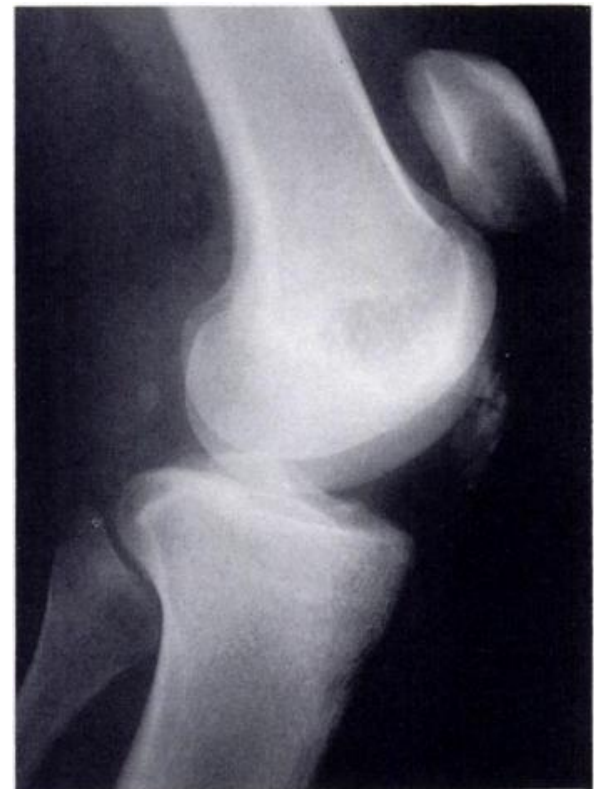

Fig. 5a

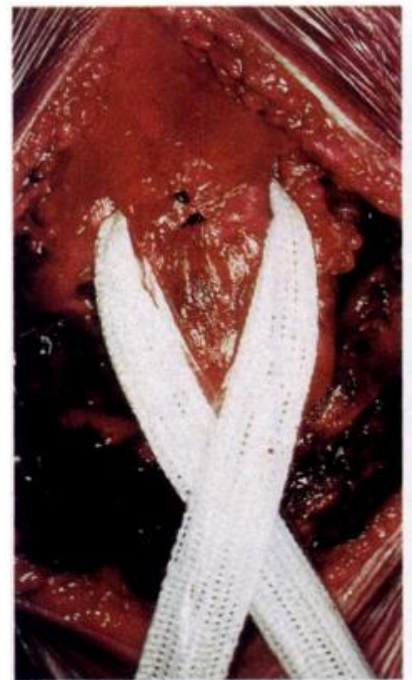

Fig. 5c

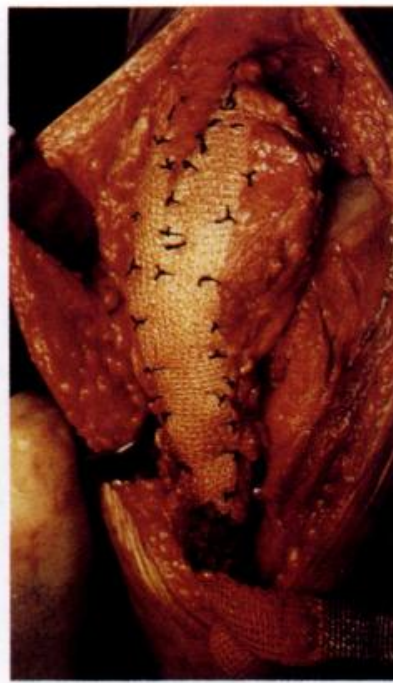

Fig. 5d

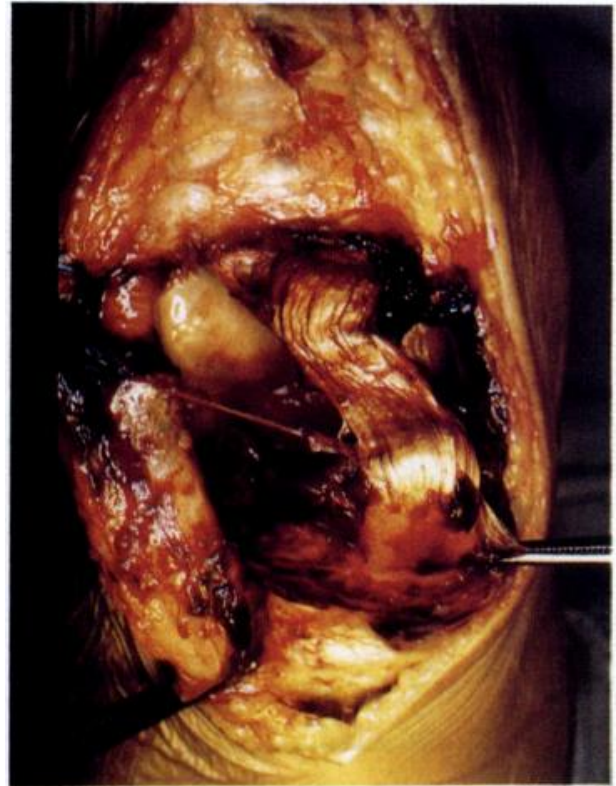

Fig. 5b

Case 2. A 50-year-old woman on haemodialysis for renal failure. Figure 5a - Preoperative radiograph. Figure 5b - The patellar tendon was avulsed from the patella. Figures $5 \mathrm{c}$ and $5 \mathrm{~d}-$ The patellar tendon was reconstructed with an artificial ligament. Figure $5 \mathrm{e}-\mathrm{Range}$ of knee movement 4.5 years after repair.

to normal activities, without a walking aid, was 10.3 weeks. There were no cases of infection, persistent effusion or rerupture.

\section{Illustrative case reports}

Case 1. A 65-year-old man had been unable to extend his right knee actively for more than two years because of an old fracture of the patella with wide separation of the fragments. The distal fragment was excised and the proximal fragment brought down by patellar tendon reconstruction. The patient regained an adequate range of motion with no extension lag. A radiograph taken five years after surgery showed the patella in the anatomical position (Fig. 4).

Case 9. A 50-year-old woman on haemodialysis for ten years because of chronic renal failure sustained a spontaneous rupture of her left patellar tendon while descending some stairs. Eight weeks after patellar tendon reconstruction she returned to normal activities with a good range of knee motion (Fig. 5).

\section{DISCUSSION}

Several procedures have been described for repair or reconstruction of the knee extensor apparatus (McLaughlin and Frances 1956; Kelikian, Riashi and Gleason 1957; Levin 1976; MacEachern and Plewes 1984), but where a synthetic substitute was used, it did not have sufficient tensile strength to allow early mobilisation (Miskew, Pearson and Pankovich 1980). This meant that cast immobilisation and a long rehabilitation were required. One advantage of the procedure that we describe is that autogenous graft material is not 
Table I. Range of movement (degrees) in 19 knees after repair of the extensor apparatus

\begin{tabular}{cll}
\hline Case & Extension & Flexion \\
\hline 1 & 0 & 160 \\
2 & 0 & 160 \\
3 & 0 & 160 \\
4 & 8 & 140 \\
5 & 0 & 150 \\
6 & 0 & 150 \\
7 & 0 & 140 \\
8 & 7 & 150 \\
9 & 0 & 160 \\
10 & 8 & 145 \\
11 & 0 & 140 \\
12 & 0 & 145 \\
13 & 0 & 145 \\
14 & 0 & 145 \\
15 & 0 & 145 \\
16 & 0 & 145 \\
17 & 0 & 140 \\
$18 \mathrm{~L}$ & 0 & 145 \\
R & 0 & 140 \\
& & \\
Mean & 1.2 & 147.6 \\
\hline
\end{tabular}

required and the remnants at the site of rupture are secured to the implant. The passage of the implant along the proximal or distal margin of the patella also serves to avoid the 'cheese-wire' effect of some other methods which use a bone tunnel through an often porotic patella. The main advantages are that the operative technique is simple, there is no need for immobilisation and rehabilitation is much quicker.
In patients with chronic renal failure, the link between tendon and bone is weakened by amyloid degeneration: conventional primary repair may fail, making some kind of augmentation essential. We had lasting good results in 12 such cases and were able to show that the implant is of sufficient strength to retain an anatomical relationship between the patella and the femoral condyle (Fujikawa, Seedhom and Wright 1983).

One or more of the authors have received or will receive benefits for personal or professional use from a commercial party related directly or indirectly to the subject of this article. In addition benefits have also been or will be directed to a research fund, foundation, educational institution, or other non-profit institution with which one or more of the authors is associated.

\section{REFERENCES}

Fujikawa K, Seedhom BB, Wright V. Biomechanics of the patellofemoral joint: Part 1: a study of the contact and the congruity of the patello-femoral compartment and movement of the patella. Eng Med $1983 ; 12: 3-11$.

Kelikian H, Riashi E, Gleason J. Restoration of quadriceps function in neglected tear of the patellar tendon. Surg Gynecol Obstet 1957; 104:200-4.

Levin PD. Reconstruction of the patellar tendon using a Dacron graft. Clin Orthop 1976; 118:70-2.

MacEachern AG, Plewes JL. Bilateral simultaneous spontaneous rupture of the quadriceps tendons: five case reports and a review of the literature. J Bone Joint Surg [Br] 1984; 66-B:81-3.

McLaughlin HL, Francis KC. Operative repair of injuries to the quadriceps extensor mechanism. Am J Surg 1956; 91:651-3.

Miskew DB, Pearson RL, Pankovich AM. Mersilene strip suture in repair of disruptions of the quadriceps and patellar tendons. J Trauma 1980; 20:867-72. 